\title{
Konsequenzen der Föderalismusreform für die Strafzumessung
}

\section{Gleichbehandlung bei der Strafzumessung trotz unter- schiedlicher Strafvollzugsgesetze?}

Die Vollzugswirklichkeit wird bislang nur sehr selten auch als ein Aspekt der Strafzumessung behandelt ${ }^{1}$. Auch bei der Anhörung zur Föderalismusreform wurde unterstellt, dass die Strafvollzugsregelungen bei der Urteilsfindung keine gewichtige Rolle spielten, und eine Berücksichtigung unterschiedlicher Normierungen den Strafrichtern zuzumuten $\operatorname{sei}^{2}$. Die Bedeutung der jeweiligen Umstände im Vollzug für die auszusprechende Strafe wird aber wegen der Notwendigkeit der Gleichbehandlung von Strafgefangenen zunehmen müssen, je mehr schon die gesetzlichen Vollzugsvorschriften voneinander abweichen. Aber eine liberalere Ausgestaltung mit großzügiger Gestattung von „Annehmlichkeiten“ ist generell weniger belastend als ein Verwahrvollzug, in dem Lockerungen als besondere Ausnahme gelten sollen. Benötigt wird daher ein diese Ungleichbehandlung ausgleichendes Element. Im Folgenden soll daher die Möglichkeit einer Berücksichtigung wesentlich unterschiedlicher Umstände im Strafvollzug bei der richterlichen Strafzumessung erörtert werden.

\section{Unterschiedlich hohe Belastung von Gefangenen}

Verschiedene Normierungen des Strafvollzugs werden voraussehbar zu deutlich uneinheitlich belastenden Bedingungen für die Strafgefangenen in den einzelnen Bundesländern führen. Das - bislang in $\ 18$ StVollzG geregelte - Recht auf nächtliche Einzelunterbringung, dem die Praxis schon jetzt nicht wirklich hinreichend gerecht wird ${ }^{3}$, soll etwa offenbar in einigen Ländern durch eine „Soll“-Vorschrift (weiter) eingeschränkt werden ${ }^{4}$. Eine unfreiwillige Zusammenlegung von Gefangenen während der Ruhezeit stellt aber angesichts der unvermeidbaren schweren gegenseitigen Beeinträchtigung der Privat- und Intimsphäre regelmäßig eine wesentlich größere Belastung der Betroffenen dar als eine Einzelunterbringung. -Auch im Bereich von Behandlungsmaßnahmen wie etwa der Entscheidung über die Unterbringung im offenen oder geschlossenen Vollzug (derzeit $\mathbb{1} 10$ StVollzG), Vollzugslockerungen (derzeit $\mathbb{1} 11$ StVollzG) oder Urlaub aus der Haft (derzeit $\ 13$ StVoll$\mathrm{zG}$ ) sind erheblich voneinander abweichende Regelungen zu erwarten. Schon bislang gab es hierbei zum Teil wesentliche Unterschiede in der Praxis der einzelnen Länder ${ }^{5}$. Es liegt nahe, dass diese Vollzugserleichterungen in Strafvollzugssystemen, die den Schutz der Allgemeinheit als gleichwertiges „Vollzugsziel“ festschreiben, grundsätzlich weniger liberal gehandhabt werden als in einem allein auf das Ziel der Resozialisierung ausgerichteten Vollzug. Die (noch) stärkere Betonung der Sicherungsaufgabe wird sich durch erhöhte Anforderungen an das Nichtvorliegen von Fluchtoder Missbrauchsgefahr hemmend auf die Gewährung solcher die Förderung der Resozialisierung bezweckender Maßnahmen auswirken. Den Gefangenen im Sicherheitsvollzug werden somit in aller Regel wesentlich weniger Lockerungen genehmigt als denen im Resozialisierungsvollzug. Dies kann zu erheblichen Verzögerungen bei der Wiedereingliederung der Betroffenen führen, da sie den persönlichen Umgang mit Personen außerhalb der Anstalt und die eigenverantwortliche Besorgung ihrer eigenen Angelegenheiten in deutlich geringerem Umfang einüben können. Neben dieser Ungleichheit hinsichtlich der Resozialisierung ist auch der reine Er- holungswert von Außenaufenthalten (besonders des Hafturlaubs) von großer Bedeutung, so dass sich prinzipiell eine großzügige Genehmigung von Vollzugslockerungen als weniger belastend für den Gefangenen darstellt. -Schließlich wird auch die Genehmigung des Besitzes von Gegenständen zur Haftraumausstattung und für die Freizeitbeschäftigung (bisher normiert in $\mathbb{S} 19$ und 70 StVollzG) voraussehbar erheblich unterschiedlicher Regelung in einzelnen Ländern unterliegen. Dabei weist schon die heutige Praxis enorme Ungleichheiten auf: Nicht selten ergehen zum gleichen Entscheidungsgegenstand gegensätzliche Entscheidungen ${ }^{6}$. Bereits bestehende voneinander abweichende Praxis-Regelungen in jüngerer Zeit (etwa Listen mit „erlaubten“ Gegenständen oder Punktekontrollsysteme ${ }^{7}$ ) und die zukünftige uneinheitliche Festlegung des Vollzugsziels lassen noch größere Abweichungen erwarten. Der Besitz eigener Sachen erhält dem Gefangenen aber grundsätzlich einen Teil seiner Persönlichkeit ${ }^{8}$. Daher ist eine restriktive Regelung dieses Bereichs generell belastender für die Gefangenen als liberalere Vorschriften. -Schon diese (wenigen) Beispiele belegen, dass bei ungleicher gesetzgeberischer und praktischer Handhabung von Vollzugsmaßnahmen deutlich unterschiedliche Belastungen auf die Strafgefangenen in einzelnen Bundesländern zukommen werden. Hierfür müsste ein Ausgleich angestrebt werden.

\section{Ausgleich durch die Strafzumessung}

Gemäß $\ 46$ Abs.1 Satz 2 StGB sind bei der Strafzumessung die Wirkungen, die von der Strafe für das künftige Leben des Täters in der Gesellschaft zu erwarten sind, zu berücksichtigen. Im Bereich der zu vollstreckenden Freiheitsstrafe wird hierdurch besonders die Resozialisierung des Betroffenen angesprochen, die nach \ 2 Satz 1 StVollzG (jedenfalls bislang) das einzige Vollzugsziel darstellt, und auf deren Beachtung jeder Strafgefangene einen grundrechtlichen Anspruch hat ${ }^{9}$. Trotz aller individuellen Unterschiede werden Strafgefangene gleichermaßen wegen Verstoßes gegen einheitliche Strafvorschriften in einem nach bundesweit übereinstimmenden Vorschriften durchgeführten Verfahren zu einer Freiheitsstrafe verurteilt. Sie sind deshalb insoweit grundsätzlich als gleichartige Gruppe anzusehen. Eine prinzipielle Ungleichbehandlung in einzelnen Bundesländern bedürfte demnach einer sachlichen Rechtfertigung, wobei die Gesetzgebungszuständigkeit der Länder allein angesichts des engen Zusammenhangs von materiellem Strafrecht, Strafverfahrensrecht und Strafvollzugsrecht ${ }^{10}$ nicht ausreichen kann. Zwar soll die Verfassungsmäßigkeit eines Landesgesetzes grundsätzlich nicht schon deshalb in Zweifel gezogen werden können, weil das Landesgesetz von verwandten Regelungen in anderen Bundesländern oder im Bund abweicht, da dies eine Folge der föderalistischen Struktur des Staates sei ${ }^{11}$. Von diesem Grundsatz muss jedoch eine Ausnahme gemacht werden, wenn das Landesgesetz unmittelbar mit Bundesgesetzen verbunden ist, d.h. wenn die zu regelnden Rechtsmaterien gegenseitig aufeinander bezogen sind oder voneinander abhängen. Dies trifft besonders bei Vollzugsgesetzen zu, da in diesen die Durchführung von Maßnahmen geregelt ist, die aufgrund anderer Gesetze angeordnet worden sind: So legt z.B. $\$ 1$ StVollzG ausdrücklich fest, dass das Gesetz den Vollzug der Freiheitsstrafe in Justizvollzugsanstalten regelt, also die praktische Ausgestaltung einer 
in anderen Gesetzen (vor allem im StGB) für ein bestimmtes Fehlverhalten angedrohten Strafsanktion. Bei solchen nicht wirklich trennbaren Zusammenhängen von Gesetzen erscheinen wesentlich voneinander abweichende Regelungen in einzelnen Bundesländern als verfassungsrechtlich problematisch. Anders als etwa bei der Frage der landesrechtlichen Behandlung von Polizeibeamten, bei Landesgesetzen über die Haftung für die Beschädigung von öffentlichem Eigentum oder bei der landesrechtlichen Gewährung von Lernmittelfreiheit für Studierende ${ }^{12}$ regeln Strafvollzugsgesetze kein unabhängiges Rechtsgebiet. Das StVollzG (oder entsprechende Landesgesetze) würde ohne materielle Strafgesetze und Strafverfahrensnormen keine Bedeutung haben: Ohne die bundeseinheitlichen Strafvorschriften und das übereinstimmend normierte Strafverfahren gäbe es keine Freiheitsstrafe, deren Vollzug geregelt werden müsste. Vom Grundsatz, dass der Landesgesetzgeber nur die Gleichbehandlung innerhalb des Landes zu beachten hat, muss zudem eine Ausnahme gelten, wenn der zu regelnde Lebenssachverhalt seiner Natur nach über die Ländergrenzen hinausgreift und eine für alle Bürger gleichermaßen gewährleistete Rechtsposition berührt ${ }^{13}$. Dies ist beim Vollzug der bundeseinheitlichen Freiheitsstrafe der Fall. Deshalb ist die Vereinbarkeit einer gesetzlich erheblich unterschiedlichen Regelung des Strafvollzugs mit dem Gleichheitssatz des Art.3 Abs.1 GG höchst zweifelhaft. Um die großen Unterschiede zwischen Resozialisierungs- und Sicherheitsvollzug ausgleichen zu können und damit eine dennoch verfassungsgemäße Regelung durch annähernd gleich hohe Belastung der Strafgefangenen zu erreichen, müsste eine Berücksichtigung der wesentlich verschiedenen Vollzugsformen ermöglicht werden. Hierfür erscheint es am sinnvollsten, die schon von der gesetzlichen Normierung her wesentlich unterschiedliche Ausgestaltung des Vollzugs bereits bei der Strafzumessung mit zu beachten. Es ist angeregt worden, die Überfüllung vieler Justizvollzugsanstalten wegen ihrer negativen Auswirkungen bei der gerichtlichen Strafzumessung zu berücksichtigen ${ }^{14}$. Dabei wären allerdings in der Praxis Probleme möglich, weil die Gefangenenzahlen schwanken und damit unsichere Grundlagen für die Bewertung der Überbelegung darstellen könnten: Bei länger einsitzenden Gefangenen und sich deutlich verändernder Belegungssituation könnte eine nachträgliche Neubemessung erforderlich werden. Solche Schwierigkeiten sind jedoch nicht bei der Abwägung der jeweiligen Wirkungen verschiedener Formen des Vollzugs in einzelnen Bundesländern zu erwarten, da sich diese Unterschiede aus den festgeschriebenen Gesetzen ergeben und daher (relativ) dauerhaft sind. Erheblich verschiedene Haftbedingungen können wertend erfasst und als strafzumessungsrelevante Tatsachen behandelt werden. Dies zeigt sich bei der Vorschrift des $₫ 51$ Abs.4 Satz 2 StGB, welche den Maßstab der Anrechnung einer vollstreckten ausländischen Freiheitsentziehung wegen derselben Tat abhängig von den jeweiligen Haftbedingungen im konkreten Land ins Ermessen des Gerichts stellt. Dabei hat der Richter das im Ausland erlittene Strafübel zu schätzen und es in ein dem inländischen Strafensystem zu entnehmendes Äquivalent umzusetzen. Er hat zu erwägen, wie schwer das Übel wiegt, das dem Verurteilten durch die ausländische Strafverfolgung widerfahren ist, und wie viel dieses von den Belastungen durch das zu fällende inländische Urteil schon vorweggenommen hat ${ }^{15}$. Somit ist vorgesehen, die deutlich unterschiedlichen Haftumstände in vielen Staaten (und teilweise auch in einzelnen Strafanstalten) bei der Anrechnung auf eine zu verhängende Strafe zu berücksichtigen, wobei verschieden hohe Belastungen zu unterschiedlichen Anrechnungsmaßstäben führen sollen. Ins Gewicht fallen dabei insbesondere (stark) über- belegte Zellen und Mängel bei der Hygiene aber auch eine unzureichende Gestattung von Bedarfs- oder Ausstattungsgegenständen und ein $(\mathrm{zu})$ geringer Umfang von Kontaktmöglichkeiten zu nahestehenden Personen ${ }^{16}$. Die gesetzlich vorgeschriebene Anrechnung erfolgt als eine Art Ausgleich dafür, dass das Verbot der Doppelbestrafung (Art.103 Abs.3 GG) grundsätzlich nicht bezüglich ausländischer Verurteilungen gilt ${ }^{17}$. Da hierfür allein jedoch bereits ein Umrechnungsmaßstab von 1:1 (entsprechend etwa $\mathbb{S} 43$ Satz 2, 51 Abs. 4 Satz 1 StGB) ausreichen würde, entspringt die Regelung der differenzierten Anrechnung offenbar zusätzlichen allgemeinen Gerechtigkeitserwägungen. Der Rechtsgedanke der Berücksichtigung wesentlich unterschiedlicher Vollzugsbedingungen lässt sich deshalb durchaus von der Anrechnung ausländischer Strafen auf die wegen deutlich voneinander abweichender gesetzlicher Regelungen $\mathrm{zu}$ erwartenden erheblich verschiedenen Umstände im Strafvollzug einzelner Bundesländer übertragen. Die Problematik ist praktisch vergleichbar. An dieser Vergleichbarkeit ändert auch nichts, dass bei der Anrechnung ausländischer Haft die konkreten Auswirkungen bereits feststehen, da dadurch lediglich die Bemessung erleichtert wird. Ansonsten geht es in beiden Fällen aber gleichermaßen um sehr unterschiedliche Haftbedingungen, die bei der Bestimmung der Höhe einer auszusprechenden Freiheitsstrafe aus Gründen der Gerechtigkeit einen gewissen Einfluss haben müssten.

\section{Zu erwartende Schwierigkeiten}

Die Berücksichtigung ungleicher regionaler gesetzlicher Regelungen des Strafvollzugs bei der Strafzumessung ist durchaus nicht unproblematisch. Grundsätzlich erscheint es im Hinblick auf den Schuldausgleich, den $\$ 46$ Abs. 1 Satz 1 StGB bezweckt ${ }^{18}$, sicherlich sinnvoll, dass härteren Vollzugsbedingungen mit einer niedrigeren Strafe begegnet wird ${ }^{19}$. Allerdings werden sich die Chancen für eine Resozialisierung von Gefangenen voraussehbar in Vollzugsformen, die schon von der gesetzlichen Zielsetzung her den Schutz der Allgemeinheit vor weiteren Straftaten als „gleichwertig“ festschreiben, wesentlich verringern. Im Sicherheits- oder Verwahrungsvollzug wird ein Gefangener wegen der Beschränkung von Resozialisierungsangeboten für das Erreichen seiner zukünftigen Lebensführung ohne Straftaten regelmäßig längere Zeit benötigen als im reinen Resozialisierungsvollzug, wodurch eine höhere Strafe notwendig erscheinen könnte. Die aus Gründen der Schuldangemessenheit erforderliche Verringerung des Strafmaßes im erheblich belastenderen Sicherheitsvollzug dürfte in der Praxis aber ohnehin wegen der Begrenzung von die Resozialisierung fördernden Angeboten zu längeren VerbüBungszeiten führen, da eine Strafrestaussetzung zur Bewährung nach $\mathbb{S} 57$ Abs.1 Satz 1 Nr.2 StGB eine günstige Prognose hinsichtlich der Verantwortbarkeit der Haftentlassung voraussetzt. Der im Vergleich zu $\mathbb{S} 56$ Abs.1 Satz 1 StGB weniger strenge Maßstab beruht nämlich darauf, dass auf den Verurteilten im Strafvollzug resozialisierend eingewirkt worden is $\mathrm{t}^{20}$, so dass die Reduzierung von Resozialisierungsangeboten insoweit zu einer Erhöhung des vollstreckten Strafanteils führen müsste ${ }^{21}$. Der theoretische Widerspruch zwischen zum Schuldausgleich niedrigerer und zwecks erfolgreicher Resozialisierung bei weniger Angeboten erforderlicher höherer Strafe wird somit voraussichtlich praktisch weitgehend durch die konkret zu verbüßende Strafzeit gelöst. Demnach dürfte die Schuldangemessenheit in aller Regel die Verurteilung zu einer geringeren Strafe im härteren Sicherheitsvollzug rechtfertigen. Die anfänglich zu erwartenden Pro- 
bleme der Bewertung des Ausmaßes der Belastungen durch verschiedene Maßnahmen in der jeweiligen Ausgestaltung des Vollzugs werden sich längerfristig erledigen: Nach einiger Zeit werden hinreichende Erfahrungen über die tatsächlichen Auswirkungen etwa der Einschränkung von Vollzugslockerungen oder der weiter erleichterten Mehrfachbelegung von Hafträumen vorliegen und sich (wie bei der Anrechnung ausländischer Freiheitsentziehung ${ }^{22}$ ) bestimmte Maßstäbe herausbilden, wie der Vollzug eines Bundeslandes im Verhältnis zu dem eines anderen hinsichtlich der Belastung von Gefangenen zu beurteilen ist. -Danach wäre es ratsam, die Berücksichtigung der wesentlich unterschiedlichen landesspezifischen Umstände im Vollzug der bundeseinheitlichen Freiheitsstrafe bei der Strafzumessung ausdrücklich gesetzlich festzuschreiben, da eine - zu deren Ausgleich nötige - ausreichende gerichtliche Beachtung innerhalb des $\mathbb{S} 46$ Abs.1 Satz 2 StGB sonst nicht immer wirklich erwartet werden kann. Bei der Erwägung des Maßes einer zu verhängenden Freiheitsstrafe hätte der Richter dann - entsprechend $\$ 51$ Abs.4 Satz 2 StGB - die jeweiligen Bedingungen im Strafvollzug des Bundeslandes zu ermitteln, in dem die Strafe vollzogen werden soll, und diese wertend (mit ausdrücklicher Begründung) in die Bemessung der Strafe einzubeziehen ${ }^{23}$. Dabei könnten allerdings in der Praxis schwere Probleme auftreten, wenn ein Gefangener später (d.h. nach der Verurteilung) in ein anderes Bundesland mit einer erheblich anderen gesetzlichen Regelung des Strafvollzugs verlegt wird. Aus Gründen der Gerechtigkeit müsste dann das rechtskräftige Urteil geändert bzw. angepasst werden. Ob dies bei einer Verlegung vom Resozialisierungs- in den Sicherheitsvollzug durch eine Strafsenkung im Rahmen des $\mathbb{} \int 59$ Nr.5 StPO geschehen könnte, ist sehr zweifelhaft: Zwar stellt die Verlegung in den belastenderen Vollzug durchaus eine nach dem Urteil eingetretene Tatsache dar, die wegen der vergleichsweise höheren Belastung eine Strafherabsetzung erlauben würde. Letztere fände aber nicht in Anwendung eines milderen Strafgesetzes statt, so dass eine Wiederaufnahme zugunsten des Verurteilten nicht möglich erscheint. $\mathbb{3} 363$ Abs.1 StPO schließt eine Wiederaufnahme zwecks anderer Strafbemessung auf Grund desselben Strafgesetzes ausdrücklich aus. Dies gilt ebenfalls im Fall der Verlegung vom Sicherheits- in den Resozialisierungsvollzug, wobei insoweit ohnehin schon kein zur Grund zur Wiederaufnahme zuungunsten des Angeklagten i.S. des $\mathbb{} 362$ StPO gegeben ist. Zusätzlich zur Festschreibung der Berücksichtigung der jeweiligen Bedingungen im Strafvollzug müsste deshalb eine Bestimmung in das Recht der Wiederaufnahme des Verfahrens aufgenommen werden, die eine nachträgliche Änderung der Strafhöhe ermöglicht, wenn der Vollzug der Strafe in einem Bundesland mit erheblich anderen gesetzlichen Vollzugsvorschriften und entsprechend unterschiedlichen Haftumständen fortgesetzt werden soll. -Die hier kurz dargestellten Schwierigkeiten zeigen auf, dass die Beachtung verschiedener Strafvollzugsgesetze bei der Strafzumessung reichlich rechtliche und tatsächliche Probleme mit sich bringen wird. Dennoch erscheint sie zum Ausgleich von mit dem allgemeinen Gleichheitssatz des Art.3 Abs.1 GG nicht zu vereinbarenden wesentlich voneinander abweichenden Vollzugsformen in einzelnen Bundesländern geboten.

\section{Konsequenzen}

Für eine unterschiedlich hohe Belastung von Strafgefangenen auf Grund verschiedener Vollzugsgesetze in den Bundesländern gibt es keine sachliche Rechtfertigung. Dass die Freiheitsstrafe in einem bestimmten Bundesland vollzogen wird, reicht allein nicht aus, um die Bestrafung tatsächlich „härter“ ausfallen zu lassen als in anderen Ländern. Ein (verfassungsrechtlich erforderlicher) Ausgleich dieser Ungleichbehandlung von Gefangenen könnte durch Berücksichtigung der regionalen Strafvollzugsbedingungen bei der Strafzumessung erfolgen. Allerdings sind hierbei viele massive Probleme in der Praxis zu befürchten, so dass der (Bundes-)Gesetzgeber gewisse Einzelheiten der praktischen Durchführung gesetzlich regeln müsste. -Es ist jedoch noch eher ratsam, die derzeitige Einheitlichkeit der gesetzlichen Strafvollzugsregelung beizubehalten, indem die Länder auf voneinander abweichende Gesetze verzichten und ein gemeinsames Strafvollzugsgesetz erschaffen. Für eine bundesweit übereinstimmende Normierung sprechen zusätzlich der nicht sinnvoll zu trennende Zusammenhang der Materien des Kriminalrechts und die frühere Einsicht der Notwendigkeit einer bundeseinheitlichen Regelung ${ }^{24}$. Trotz derzeitiger Bemühungen um Alleingänge in einzelnen Ländern (wie etwa dem Niedersächsischen Justizvollzugsgesetz (NJVollzG)) erscheint ein Aufhalten der Zersplitterung des Strafvollzugs, die ein eindeutiges Zeichen für eine völlig vernunft- und sinnwidrige Gesetzgebung darstellt, noch möglich.

Der Autor ist Assessor in Bremen und Mitautor der 5.Auflage des Alternativkommentars zum Strafvollzugsgesetz (AK-StVollzG).

\section{Fußnoten}

1 Ähnlich Schott, ZfStrVo 2003, 195, 198.

2 Vgl. Mävers, DRiZ 2006, 214.

3 Vgl. etwa Kellermann/Köhne, in AK-StVollzG, 5.Aufl.2006, \18 Rdnr.3; Böhm, in Schwind/Böhm/Jehle, StVollzG, 4.Aufl.2005, \18 Rdnr.2.

4 Kritisch zum Gesetzesentwurf BR-Drs. 923/03 etwa Kellermann/Köhne, (Fn.5); Calliess/Müller-Dietz, StVollzG, 10.Aufl.2005, \18 Rdnr.1; Mushoff, FoR 2004, 20 ff.; Kretschmer, NStZ 2005, 251, 255.

5 Näher hierzu etwa Feest/Lesting, ZfStrVo 2005, 76, 79 ff.; Lesting, in AK-StVollzG, 5.Aufl.2006, \10 Rdnr.6, \11 Rdnr.2 und $\$ 13$ Rdnr.2.

6 Vgl. Calliess/Müller-Dietz, (Fn.4), \ 70 Rdnr.3; Köhne, ZfStrVo 2005, 280.

7 Hierzu etwa Kellermann/Köhne, (Fn.5), \ 19 Rdnr.3; zur „REFA-Haftraumkontrolle" Köhne, ZfStrVo 2002, 345 f.

8 Ähnlich Böhm, (Fn.3), \19 Rdnr.1.

9 Vgl. BVerfGE 98, 169, 200 = NJW 1998, 3337.

10 Hierzu etwa Müller-Dietz, ZfStrVo 2005, 38, 39; Köhne, ZRP 2006, 195 f.

11 Vgl. etwa BVerfGE 17, 319, 331; BVerfGE 42, 20, 27; auch BVerwGE 102, 142, $148 \mathrm{f}$. = NJW 1997, 2465, 2467.

12 Vgl. die Sachverhalte der in Fn.11 genannten Entscheidungen.

13 Vgl. BVerfGE 33, 303, 352.

14 Vgl. Schott, (Fn.1), 197 ff.

15 Vgl. etwa BGHSt 30, 282, 283; BGH NStZ 1986, 312, 313.

16 Vgl. etwa OLG Zweibrücken GA 1993, 126; OLG Düsseldorf StV 1995, 426, 427; OLG Celle NStZ 1998, 137, 138; LG Zweibrücken NStZ 1988, 71; LG Köln NStE Nr.20 zu $\ 51$ StGB; LG Zweibrücken MDR 1995, 84; LG Zweibrücken MDR 1997, 279, 280.

17 Näher hierzu etwa Müller-Dietz, in FS-Salger, 1995, S. 105, 107 f.

18 Eingehend zur „Schuld“ im Rahmen der Strafzumessung Hörnle, JZ 1999, 1080 ff.

19 Ähnlich Schott, (Fn.3), 197 zum Schuldausgleich in Zeiten hoher Überbelegung von Gefängnissen.

20 So BGH NStZ-RR 2003, 200, 201; Stree, in Schönke/Schröder, StGB, 27.Auflage 2006, \$5 57 Rdnr.10.

21 Worauf auch Caspari, DRiZ 2006, 142 hinweist.

22 Vgl. etwa Tröndle/Fischer, StGB, 54.Aufl.2007, \51 Rdnr.19; Lemke, in NKStGB Band 1, 2.Aufl.2005, $\ 51$ Rdnr.41; Franke, in MüKo-StGB Band 1, 2003, $\mathbb{S}$ 51 Rdnr.24f.

23 Caspari, (Fn.21) bezeichnet eine Strafzumessung unter Beachtung der Vollzugsregelungen eines anderen Bundeslandes als besonders erschwerend.

24 Die 1961 zum Erlass der einheitlichen Dienst- und Vollzugsordnung (DVollzO) durch die Länder führte. 